\title{
The evolution of nova shells
}

\section{Tappert*}

Instituto de Física y Astronomía, Universidad de Vaparaíso, Chile

E-mail: claus.tappert@uv.cl

\section{E. Arancibia}

Instituto de Física y Astronomía, Universidad de Vaparaíso, Chile

E-mail: eduardo.arancibia@alumnos.uv.cl

\section{Schmidtobreick}

European Southern Observatory, Santiago, Chile

E-mail: Ischmidt@eso.org

\section{N. Vogt}

Instituto de Física y Astronomía, Universidad de Vaparaíso, Chile

E-mail: nikolaus.vogt@uv.cl

\section{A. Ederoclite}

Centro de Estudios de Física del Cosmos de Aragón, Teruel, Spain

E-mail: aederocl@cefca.es

\section{Vučković}

Instituto de Física y Astronomía, Universidad de Vaparaíso, Chile

E-mail: maja.vuckovic@uv.cl

\section{A. R. M. Ribeiro}

Univeridade de Aveiro, Portugal

E-mail: valerio.alipio.ribeiro@gmail.com

In order to investigate the luminosity and expansion evolution of nova shells, we have started a project to reobserve the novae collected in the Downes et al. (2001) catalogue on shell luminosities. In the present article, we discuss potential problems of the Downes et al. work and conduct a first, very preliminary, analysis of the expansion evolution of our first set of shell data.

The Golden Age of Cataclysmic Variables and Related Objects IV

11-16 September, 2017

Palermo, Italy

\footnotetext{
${ }^{*}$ Speaker.
} 


\section{Introduction}

In order to study the long-term effect of the nova eruption on the underlying cataclysmic binary $(\mathrm{CV})$, it is important to compare the properties of post-novae with those of the general CV population $[8,29]$. The smoking gun for establishing a CV as a former nova is the presence of a nova shell. In the recent years, such shells have been detected around the dwarf novae Z Cam and AT Cnc [21, 23], the nova-like V1315 Aql [17], and the probable intermediate polar J17014 $[25,16]$. The ages of these shells have been determined to $\geq 1300 \mathrm{yr}, 400 \mathrm{yr}, 120 \mathrm{yr}$ and $580 \mathrm{yr}$, respectively (for Z Cam and AT Cnc, see [22, 24]). On the other hand, a number of searches for shells signatures in a total of slightly more than $110 \mathrm{CVs}$ were unsuccessful $[17,18,19,15]$. Thus, either those CVs are much older (in the sense of time after the nova eruption) post-novae than the four cases above, or their shells became undetectable much faster. Cohen [1] and Downes \& Duerbeck [4] in their investigations on known post-novae detected shells in roughly 50\% of their targets. Which, then, are the parameters that determine for how long a nova shell can be reasonably detected?

\section{The luminosity evolution of nova shells}
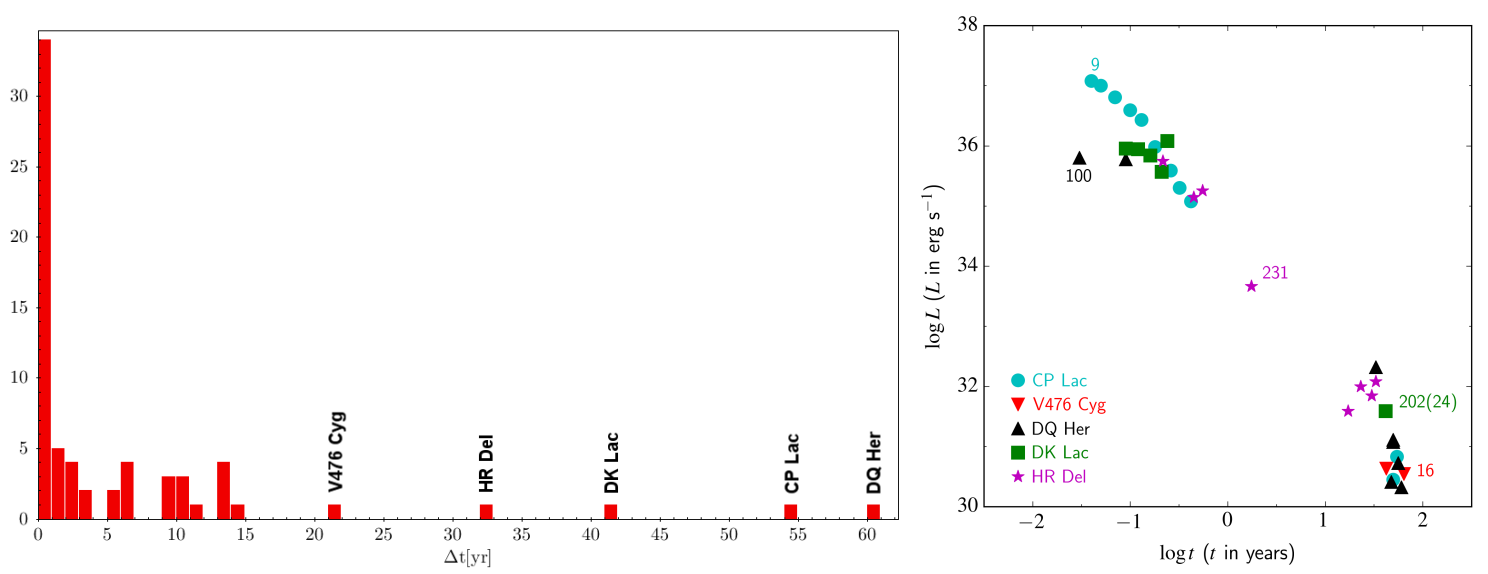

Figure 1: Left: Histogram of the time range $\Delta t$ spun by the data points of an individual nova in DDD01. The five novae with the largest time range are labelled. Right: Comparison of the luminosity evolution of those five novae. The numbers state the decline time $t_{3}$ for a given nova; for DK Lac, two very different values are reported (DDD01, [28]).

Downes et al. [5] (hereafter DDD01) investigated the hydrogen $(\mathrm{H} \alpha$ and $\mathrm{H} \beta$ ) and [OIII] luminosities of a large sample of nova shells, distinguishing novae of different speed class according to the times $t_{2}$ and $t_{3}$ in which the nova declines by two and three magnitudes after maximum brightness, respectively. They derive several nominal relations for the luminosity of the lines as a function of time after eruption, $L(t)$. In particular, they find two different relations per speed class, in the form of a slow early and a steep late decline, with the breakpoint between the two relations typically at a shell age $100 \mathrm{~d} \leq t \leq 3 \mathrm{yr}$. While this study represents a valuable step in the right direction, and, in fact, remains the only comprehensive work on the brightness evolution of nova shells to date, there are a number of potential problems associated with it. 
1. Sample size: not all speed classes count with a sufficiently even distribution of data points along the age axis. The most significant example is that of the $\mathrm{H} \alpha$ emission in slow novae, which is dominated by the behaviour of a single, extragalactic, nova (figure 10 in DDD01).

2. Grouping: the data set predominantly consists of novae with one single measurement of the shell luminosity. Only five novae have a data point distribution that spans more than $20 \mathrm{yr}$ (Fig. 1, left).

3. A single parameter: the decline rate of novae is roughly correlated with the ejection energy and thus the velocity of the ejected material (e.g., [13]). It is thus very reasonable to assume that also $L(t)$ depends on $t_{3}$. However, a number of other parameters come to mind that are also likely to affect the brightness of the shell, e.g. the structure of the surrounding interstellar medium, or dust formation in the shell material. The validity of deriving $L(t)$ from different novae of the same speed class (see point 2), depends critically on $t_{3}$ being the dominant parameter. In the right plot of Fig. 1 we compare the behaviour of the five novae with the largest time range in DDD01. This includes systems of very different speed classes. Nevertheless, especially at the later stages $(\log t>0)$, the behaviour appears to be consistent for all novae, regardless of this parameter.

The gist of the points above is that it appears desirable to extend and re-analyse the DDD01 data. Our plan of attack is the following: first, take $\mathrm{H} \alpha$ narrow-band photometry of all novae in the DDD01 paper to check for the presence of extended shells and flux-calibrated spectroscopy to measure emission line fluxes, and second, analyse the data with respect to possible correlations of above mentioned parameters.

\section{First preliminary results: deceleration}

Our main purpose is to extend the DDD01 data. This will yield a second data point in the $L-t$ diagram for all novae in DDD01, and thus allow for an analysis of the shell decline rate for individual systems, instead of having to rely on fitting a sample of different systems grouped together by a specific parameter. However, the nature of our observations allows for an investigation of additional phenomena, e.g. the evolution of the expansion. This bears importance for the distance determination of novae, as well as for the age-dating ancient novae (e.g., [24]).

Duerbeck [6] parametrized the expansion of the nova shell as

$$
r(t)=c_{0}+c_{1} t+0.5 c_{2} t^{2}
$$

with $r(t)$ being the radius of the shell at time $t, c_{0}=0$ the initial size of the shell, $c_{1}$ the initial expansion rate, and $c_{2}$ the deceleration parameter. He used this ansatz to fit the expansion data on four classical novae and found significant deceleration for all four of them.

From July 23 to 25, 2017, we observed a sample of classical novae from DDD01 on La Silla Observatory, using the New Technology Telescope (NTT) with the ESO Faint Object Spectrograph and Camera (EFOSC2). We used an $\mathrm{H} \alpha$ on-band (\#692) as well as an off-band (\#698) narrow-band filter. Fig. 2 shows the resulting difference (on-band minus off-band) images for the nova shells of V842 Cen (Nova Cen 1986), HR Del (Nova Del 1967) and FH Ser (Nova Ser 1970). 

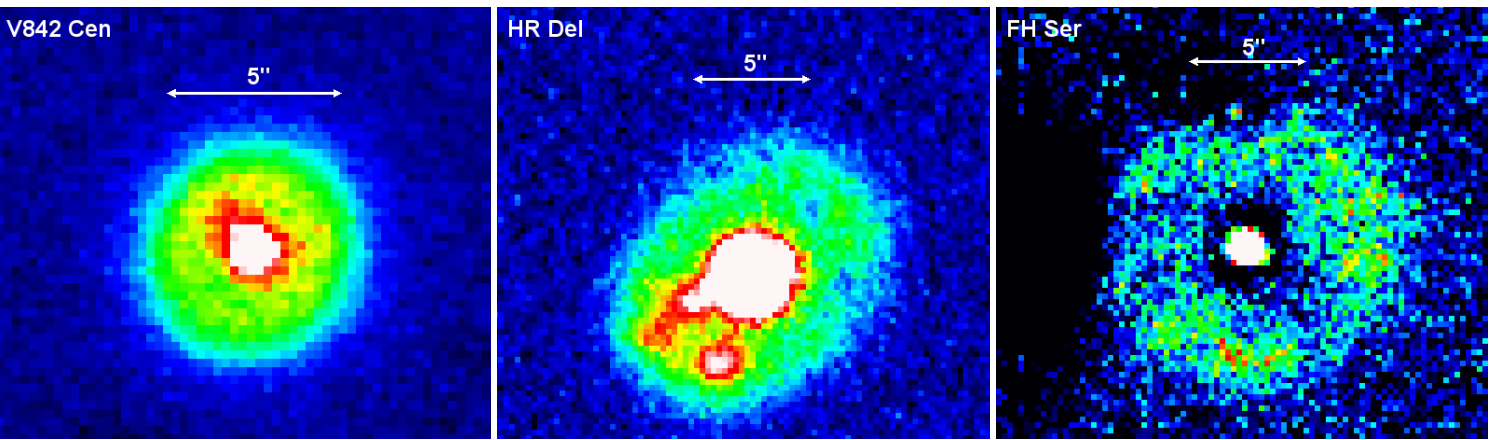

Figure 2: EFOSC H $\alpha$ data on the classical novae V842 Cen (left), HR Del (middle) and FH Ser (right).

Table 1: Shell radii $r$ for the three novae at a given point $t$ in time.

\begin{tabular}{llll}
\hline object & $t$ [yr] & $r$ [arcsec] & reference \\
\hline V842 Cen & 8.25 & 0.8 & {$[10]$} \\
& 11.33 & 3.0 & {$[4]$} \\
& 25.22 & 3.6 & {$[30]$} \\
& 30.66 & $5.2(5)$ & this work \\
HR Del & 13.76 & 1.85 & {$[12]$} \\
& 25.01 & 2.75 & {$[26]$} \\
& 26.12 & 5.75 & {$[27]$} \\
& 29.45 & 3.78 & {$[9]$} \\
& 29.83 & 4.35 & {$[4]$} \\
& 30.84 & 4.9 & {$[4]$} \\
& 34.58 & 8 & {$[14]$} \\
& 49.99 & $7.7(3)$ & this work \\
FH Ser & 12.46 & 2.0 & {$[2]$} \\
& 14.44 & 1.85 & {$[20]$} \\
& 19.50 & 2.65 & {$[7]$} \\
& 23.52 & 3.5 & {$[27]$} \\
& 26.05 & 4.5 & {$[3]$} \\
& 27.20 & 3.5 & {$[11]$} \\
& 47.40 & $7.0(6)$ & this work \\
\hline
\end{tabular}

When talking about the size of a shell, one has to define which part of the surface brightness distribution is to be measured. The general consensus seems to be that the limiting value is taken as one sigma above the sky background. However, this also is not without problems, as it strongly depends on the quality of the data. This is, e.g., discussed in [4] with respect to the discrepancy of the shell size derived in the high-quality data of [27] with their own data and those of other authors. Such ambiguity can be resolved once the shell shows localized inhomogeneities, whose motion can be followed more precisely, but this only becomes feasible at the later stages of the expansion. 


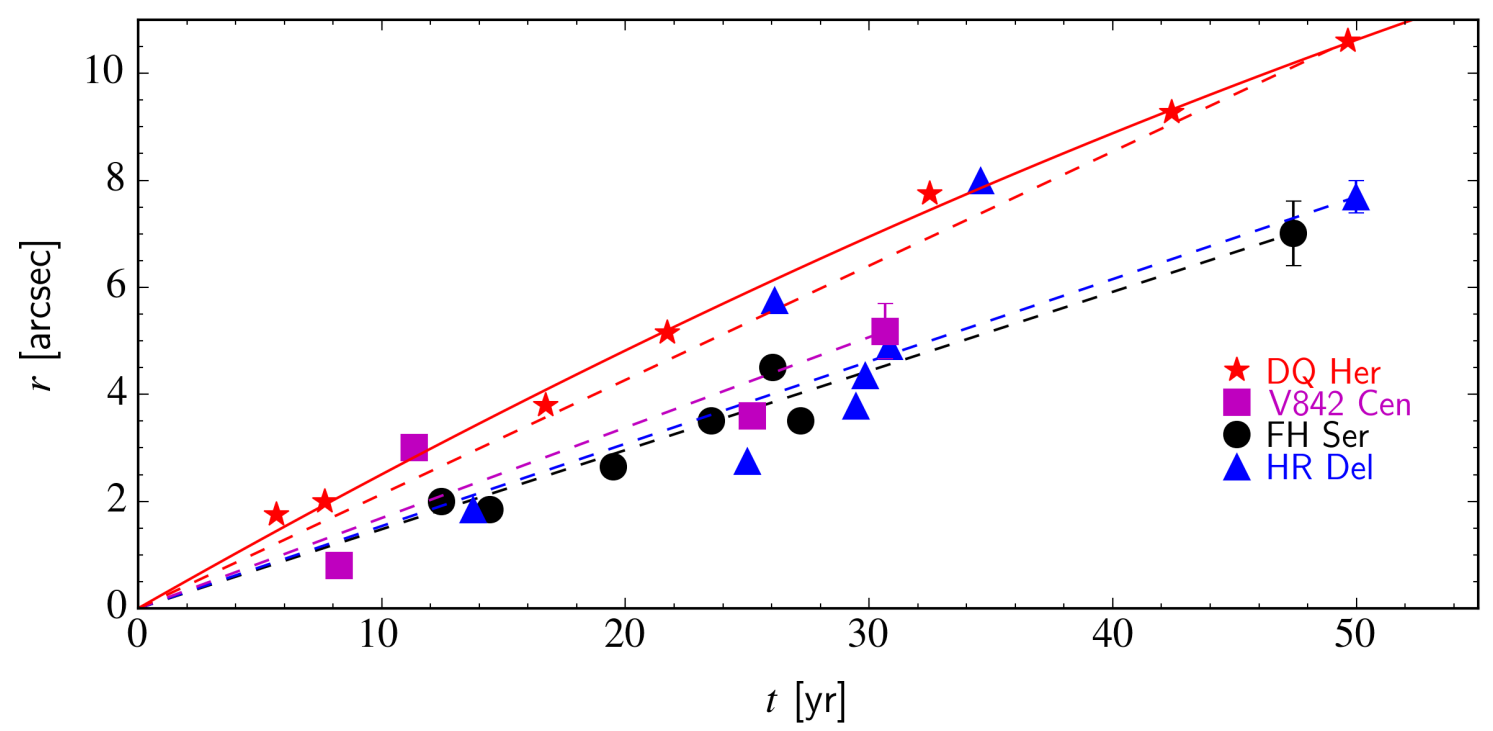

Figure 3: Shell radii $r$ versus time $t$ for the three observed novae and data on DQ Her taken from [6]. The dashed lines represent a linear evolution from the zero point to the final data point. The solid line represents the deceleration fit to DQ Her from [6].

In our preliminary analysis on the three novae, we used the one sigma above background criterion. In this way, we find the shell sizes corresponding to the semi-axes to 5.2(5) $\times 5.2(1) \operatorname{arcsec}$, 7.7(3) $\times 6.6(4)$ arcsec and 7.0(6) $\times 6.2(3)$ arcsec, for V842 Cen, HR Del and FH Ser, respectively, with the errors reflecting the differences of the measurements of the semi-axes to both sides of the central star.

In order to check for a possible deceleration, we plot our values of the major semi-axes together with those of other authors (Table 1) in Fig. 3. For a first rough test, we compare the distribution of the data with a linear expansion, as defined by the zero point $(0,0)$ and the result from our EFOSC2 data. Clearly, within the scatter, all data are consistent with zero deceleration. Duerbeck [6] finds in his data that the deceleration is inversely related to the expansion velocity. For comparison, we also show the data for the nova with the slowest expansion in the Duerbeck sample, DQ Her, which consequently also counts with the smallest deceleration parameter ${ }^{1}$. It is evident that (a) DQ Her has a significantly higher expansion rate than the other three novae, and (b) that the data are not consistent with a linear expansion, but require deceleration. We also remark that the careful re-analysis by [6] of previous data yields much less scatter than for our systems. In conclusion, we note that while our result is consistent with the tendency that nova shells with a slower initial expansion velocity show smaller deceleration, a more thorough analysis of all the available data is necessary.

\footnotetext{
${ }^{1}$ Note that there is a mistake in [6] with respect to the presentation of the deceleration parameters (or the braking parameter that he uses in his table 2), in that both the deceleration parameter and the sign of the second term in the deceleration equation are positive. This, however, yields acceleration.
} 


\section{Summary}

We have presented the outline of a project to investigate the luminosity evolution of nova shells. As an example of the first data taken in this project, $\mathrm{H} \alpha$ narrow-band images show the resolved shells of the novae V842 Cen, HR Del and FH Ser. A preliminary analysis of the data yields the sizes of the shells. In comparison with previous data we find no evidence for deceleration in either of the three systems. We suspect that the scatter in the radii data taken from several different sources impedes the detection of deceleration, which, as we argue, is also expected to be comparatively small. A careful re-analysis of previous data will be needed for a more uniform determination of shell sizes.

\section{Acknowledgments}

CT, EA, MV and NV acknowledge support by Fondecyt project 1170566.

\section{References}

[1] J. G. Cohen, 1985, Nova shells. II - Calibration of the distance scale using novae, ApJ, 292, 90

[2] J. G. Cohen, A. J Rosenthal, 1983, Nova shells, ApJ, 268, 689

[3] M. della Valle, R. Gilmozzi, A. Bianchini, H. Esenoglu, 1997, Study of nova shells. II. FH Serpentis 1970 and $Q U$ Vulpeculae 1984, nebular expansion, parallax and luminosity, A\&A, 325, 1151

[4] R. A. Downes, H. W. Duerbeck, 2000, Optical Imaging of Nova Shells and the Maximum Magnitude-Rate of Decline Relationship, AJ, 120, 2007

[5] R. A. Downes, H. W. Duerbeck, C. E. Delahodde, 2001, Luminosities of [O III] and Hydrogen Balmer lines in nova shells years and decades after outburst, Journ. Astron. Data, 7, 6

[6] H. W. Duerbeck, 1987, The interaction of nova shells with the interstellar medium, Astroph. Space Sci., 131, 461

[7] H. W. Duerbeck, 1992, The nebular expansion parallax and the luminosity of nova FH Ser, Acta Astron., 42, 85

[8] A. Ederoclite, et al., these proceedings

[9] D. J. Harman, T. J. O’Brien, 2003, Hubble Space Telescope imaging and ground-based spectroscopy of old nova shells - II. The bipolar shell of the slow nova HR Del, MNRAS, 344, 1219

[10] C. D. Gill, T. J. O’Brien, 1998, Deep optical imaging of nova remnants: a southern sky sample, MNRAS, 300, 221

[11] C. D. Gill, T. J. O'Brien, 2000, Hubble Space Telescope imaging and ground-based spectroscopy of old nova shells - I. FH Ser, V533 Her, BT Mon, DK Lac and V476 Cyg, MNRAS, 314, 175

[12] L. Kohoutek, 1981, Direct photography of the nebular remnant of Nova Delphini 1967, MNRAS, 196, P87

[13] M. Livio, 1992, Classical novae and the extragalactic distance scale, ApJ, 393, 516

[14] M. Moraes, M. Diaz, 2009, HR Del Remnant Anatomy Using Two-Dimensional Spectral Data and Three-Dimensional Photoionization Shell Models, AJ, 138, 1541 
[15] A. Pagnotta, D. Zurek, 2016, Non-detection of nova shells around asynchronous polars, MNRAS, 458, 1833

[16] S. B. Potter, D. A. H. Buckley, 2018, Discovery of spin-modulated circular polarization from IGR J17014-4306, the remnant of Nova Scorpii 1437 AD, MNRAS, 473, 4692

[17] D. I. Sahman, V. S. Dhillon, C. Knigge, T. R. Marsh, 2015, Searching for nova shells around cataclysmic variables, MNRAS, 451, 2863

[18] L. Schmidtobreick, M. Shara, C. Tappert, A. Bayo, A. Ederoclite, 2015, On the absence of nova shells, MNRAS, 449, 2215

[19] L. Schmidtobreick, et al., 2018, in preparation

[20] W. C. Seitter, H. W. Duerbeck, 1987, An Atlas of Nova Shells, in: RS Ophiuchi (1985) and the Recurrent Nova Phenomenon, Proceedings, M. F. Bode (ed.), Utrecht: VNU Science Press, p.71

[21] M. M. Shara, C. D. Martin, M. Seibert, et al., 2007, An ancient nova shell around the dwarf nova Z Camelopardalis, Nature, 446, 159

[22] M. M. Shara, T. Mizusawa, D. Zurek, et al., 2012, The Inter-eruption Timescale of Classical Novae from Expansion of the Z Camelopardalis Shell, ApJ, 756, 107

[23] M. M. Shara, T. Mizusawa, P. Wehinger, 2012, AT Cnc: A Second Dwarf Nova with a Classical Nova Shell, ApJ, 758, 121

[24] M. M. Shara, L. Drissen, T. Martin, A. Alarie, F. R. Stephenson, 2017, When does an old nova become a dwarf nova? Kinematics and age of the nova shell of the dwarf nova AT Cancri, MNRAS, 465, 739

[25] M. M. Shara, K. Ilkiewicz, J. Mikolajewska, et al., 2017, Proper-motion age dating of the progeny of Nova Scorpii AD 1437, Nature, 548, 558

[26] A. J. Slavin, T. J. O'Brien, J. S. Dunlop, 1994, The Structure of the Remnant of HR Delphini, MNRAS, 266, L55

[27] A. J. Slavin, T. J. O’Brien, J. S. Dunlop, 1995, A deep optical imaging study of the nebular remnants of classical novae, MNRAS, 276, 353

[28] R. J. Strope, B. E. Schaefer, A. A. Henden, 2010, Catalog of 93 Nova Light Curves: Classification and Properties, AJ, 140, 34

[29] C. Tappert, D. Barria, D., I. Fuentes Morales, et al., 2016, Life after eruption - VI. Recovery of the old novae EL Aql, V606 Aql, V908 Oph, V1149 Sgr, V1583 Sgr and V3964 Sgr, MNRAS, 462, 1371

[30] T. Tomov, E. Swierczynski, M. Mikolajewski, K. Ilkiewicz, 2015, SALT observations of southern post-novae, A\&A, 576, A119

\section{DISCUSSION}

SOLEN BALMAN: What exactly is the acceleration or deceleration equation you are using? Note that you have first an energy and momentum conserving free expansion phase followed by a slow down phase and a radiative phase, the $r(t)$ should be calculated accordingly.

CLAUS TAPPERT: We use a simple parametrized acceleration / deceleration equation in the form of a polynomial of second order, as introduced by [6]. All physics are hidden in the parameters of this equation, which will have to be compared to proper models once a sufficiently large sample of observed expansion rates is available. 